\title{
Publisher's Note: Identification of Nuclear Effects in Neutrino-Carbon Interactions at Low Three-Momentum Transfer [Phys. Rev. Lett. 116, 071802 (2016)]
}

P. A. Rodrigues, J. Demgen, E. Miltenberger, L. Aliaga, O. Altinok, L. Bellantoni, A. Bercellie, M. Betancourt, A. Bodek, A. Bravar, H. Budd, T. Cai, M. F. Carneiro, J. Chvojka, J. Devan, S. A. Dytman, G. A. Díaz, B. Eberly, M. Elkins, J. Felix, L. Fields, R. Fine, A. M. Gago, R. Galindo, H. Gallagher, A. Ghosh, T. Golan, R. Gran, D. A. Harris, A. Higuera, K. Hurtado, M. Kiveni, J. Kleykamp, M. Kordosky, T. Le, J. R. Leistico, A. Lovlein, E. Maher, S. Manly, W. A. Mann, C. M. Marshall, D. A. Martinez Caicedo, K. S. McFarland, C. L. McGivern, A. M. McGowan, B. Messerly, J. Miller,

A. Mislivec, J. G. Morfín, J. Mousseau, T. Muhlbeier, D. Naples, J. K. Nelson, A. Norrick, Nuruzzaman, J. Osta, V. Paolone, C. E. Patrick, G. N. Perdue, M. A. Ramirez, R. D. Ransome, H. Ray, L. Ren, D. Rimal, D. Ruterbories, H. Schellman, D. W. Schmitz, C. J. Solano Salinas, N. Tagg, B. G. Tice, E. Valencia, T. Walton, J. Wolcott, M. Wospakrik, G. Zavala, and D. Zhang

(MINERvA Collaboration)

(Received 30 October 2018; published 15 November 2018)

DOI: 10.1103/PhysRevLett.121.209902

This paper was published online on 17 September 2016 with an error in Fig. 2. Figure 2 has been replaced as of 8 November 2018. The figure is incorrect in the printed version of the journal; therefore, for the benefit of the print readership, the figure is replicated below.



FIG. 1. The double-differential cross section $d^{2} \sigma / d E_{\text {avail }} d q_{3}$ in six regions of $q_{3}$ is compared to the GENIE 2.8.4 model with reduced pion production (small dot line), the same with RPA suppression (long-dashed), and then combined with a QE-like $2 p 2 h$ component (solid). The $2 p 2 h$ component is shown separately as a shaded region. GENIE predicts events with zero available energy (all neutrons in the final state); as is done here in order to compare to data, the cross section must be summed including the spike at zero to the edge of the the first bin in each $q_{3}$ range to produce an average cross section. 